\title{
Evaluación del efecto del plasma rico en plaquetas en diferentes tiempos y concentraciones sobre la viabilidad de fibroblastos de ligamento periodontal y osteoblastos
}

\author{
Evaluation of Platelet-Rich Plasma Effect at Different Times and Concentrations on Periodontal Ligament \\ Fibroblast and Osteoblast Viability
}

\author{
Adriana Paola Acosta Gómez a \\ Pontificia Universidad Javeriana, Colombia \\ adriana.acosta@javeriana.edu.co \\ Sandra Janeth Gutiérrez Prieto \\ Pontificia Universidad Javeriana, Colombia \\ Maria Alexandra Bedoya Mejía \\ Pontificia Universidad Javeriana, Colombia \\ Dabeiba Adriana García Robayo \\ Pontificia Universidad Javeriana, Colombia \\ Ximena Edilia Moreno Serrano \\ Pontificia Universidad Javeriana, Colombia
}

DOI: https://doi.org/10.11144/Javeriana.uo36-76.eepr Redalyc: http://www.redalyc.org/articulo.oa? id $=231252657005$

Fecha de recepción: 30/06/16

Fecha de aprobación: 17/06/17

\section{Resumen:}

Antecedentes: Los factores de crecimiento utilizados en salud se pueden obtener de una fuente autóloga de primera generación llamada plasma rico en plaquetas (PRP). La diversidad de protocolos para prepararlos genera resultados variables en cuanto al tiempo entre la activación del PRP y sus efectos sobre la proliferación y viabilidad celular. Objetivo: Evaluar proliferación y viabilidad celular de Abroblastos de ligamento periodontal y osteoblastos tratados con PRP en diferentes concentraciones y tiempos de aplicación. Métodos: Se cultivaron líneas celulares de abroblastos y osteoblastos y se preparó el PRP de sangre venosa de un adulto sano mediante centrifugación, seguido de activación con $\mathrm{CaCl}_{2}$ al $10 \%$. El efecto sobre la proliferación de las líneas celulares tras la aplicación de PRP y plasma pobre en plaquetas al $1 \%$, al $3 \%$ y al $5 \%$ se evaluó a las $0,12,24,48$ y 72 horas después de su activación mediante MTS. El grupo control consistió en cultivos sin tratamiento. Los datos se analizaron mediante las pruebas de Chi cuadrado, Fischer y McNemar. Resultados: Se observó un aumento de la viabilidad en células tratadas con PRP 24 horas después de su activación en una concentración del 5\%. El ensayo de viabilidad celular mostró diferencias estadísticamente signiacativas entre el grupo experimental y el grupo control $(\mathrm{p}=0,05)$. Conclusión: Los cultivos de fibroblastos y osteoblastos mostraron una tendencia a mayor viabilidad 24 horas después de a la activación con PRP al $5 \%$.

Palabras clave: cultivos celulares, Abroblasto de ligamento periodontal, in vitro, osteoblasto, plasma rico en plaquetas, proliferación celular, viabilidad celular.

Áreas temáticas: biología molecular; periodoncia.

\begin{abstract}
:
Background: rowth factors used in health treatments can be obtained from a Arst-generation source called platelet-rich plasma. «e variety of protocols to prepare PRP produces variable results regarding PRP activation time and its effects on cell proliferation and viability. Purpose: To evaluate proliferation and cell viability of periodontal ligament Abroblasts and osteoblasts stimulated with PRP in several concentrations and times a éer PRP activation. Methods: An in vitro study was carried out using periodontal ligament abroblast and osteoblast cell cultures. PRP from venous blood of a healthy adult was prepared through centrifugation and activated with $10 \% \mathrm{CaCl}_{2}$. «e effect on cell proliferation aÁerapplication of $1 \%, 3 \%$, and $5 \%$ PRP and platelet-poor plasma was evaluated at $0,12,24,48$, and 72 hours a exeractivation through MTS. Æe control group consisted of culture that did not receive any treatment. Data were analyzed using Chi square, Fisher, and McNemar tests. Results: Æe cell viability assay showed statistically signiacant differences between the experimental and the control groups. Cell viability increased in cells treated with 5\% PRP 24 hours a exer activation $(\mathrm{p}=0.05)$. Conclusions: Fibroblast and osteoblast cell

a Autora de correspondencia. Correo electrónico: adriana.acosta@javeriana.edu.co
\end{abstract}


lines tended to be more viable 24 hours after activation with $5 \%$ PRP.

Keywords: cell cultures, cell proliferation, cell viability, in vitro, osteoblast, platelet-rich plasma, periodontal ligament fibroblast. Thematics fields: molecular biology; periodontics.

\section{INTRODUCCIÓN}

En la enfermedad periodontal, la interacción entre los diferentes microorganismos patógenos y la respuesta inmune del huésped susceptible es una de las causas principales de la afección del periodonto de protección y de inserción. Dicha interacción produce secuelas en el epitelio de unión, el ligamento periodontal, el cemento radicular y el hueso alveolar $[1,2,3]$. De ahí que el objetivo fundamental del tratamiento sea lograr la regeneración de estos tejidos $[4,5,6,7,8,9,10,11,12]$, un proceso biológico complejo que involucra proteínas de la matriz y procesos de crecimiento, migración y diferenciación celular, en los cuales están involucrados diferentes factores de crecimiento $[6,7,8,9,10,11,13]$. Los derivados sanguíneos que contienen plaquetas son una fuente importante de factores de crecimiento para los cuales a la fecha no existe consenso con respecto a la terminología, la caracterización y la clasiacación $[4,7,8,9,14,15,16,17,18,19,20,21,22$, $23,24,25]$.

Los factores de crecimiento obtenidos de las plaquetas se utilizan en diferentes áreas de la medicina y la odontología desde su primera aplicación en 1980 para el tratamiento de úlceras cutáneas [14,16, 23, 26, 27, $28,29,30]$. Estos se concentran en el plasma rico en plaquetas (PRP), un derivado sanguíneo concentrado de primera generación que se obtiene mediante centrifugación de sangre total y se caracteriza por poseer una concentración cuatro a seis veces mayor que los valores normales de las plaquetas por mililitro [8,16, 30, 31]. Se han descrito diversas aplicaciones del PRP en ołálmología, otorrinolaringología, odontología, medicina estética y regenerativa; sin embargo, su utilidad no se ha demostrado completamente debido a la heterogeneidad en los diseños experimentales y, por ende, los resultados no son comparables [16, 23, 26, 27, 28, 29, 30, 31, 32, 33]. La gran cantidad de factores de crecimiento contenidos en los gránulos plaquetarios y su capacidad de síntesis de novo de proteínas, así como su actividad microbicida y moduladora de la inÁamación, favorecen la proliferación celular y la síntesis de matriz extracelular. Así, se promueve la cicatrización y la reparación de heridas y otras lesiones tisulares $[3,4,5,6,7,8,9]$. Marx en 1986 fue el primero en describir el uso del PRP en la colocación de injertos óseos en cirugía maxilofacial y desde esa época se le atribuyeron propiedades fundamentadas en la capacidad que tiene de incrementar la regeneración ósea al ser utilizado junto con injertos de hueso [31]. No obstante, su uso ha sido inconstante y los resultados variables, debido a la diversidad en las técnicas de preparación y utilización.

Los factores de crecimiento identiacados en el PRP son el factor de crecimiento derivado de plaquetas (PDGF A y B en sus diferentes formas), el factor de crecimiento transformante beta (TGF- $\beta$ ), el factor de crecimiento semejante a la insulina, el factor de crecimiento Abroblástico, el factor de crecimiento endotelial y el factor de crecimiento epidérmico [7, 9, 16, 18, 23, 24, 30, 31, 34, 35, 36]. Estos se liberan al momento de su procesamiento mediante cloruro cálcico o trombina [15, 30, 37, 38, 39]. Aunque se ha reportado que el tiempo de aplicación del PRP debe ser antes de las 24 horas, no se conoce el tiempo ideal posterior a su procesamiento para la aplicación en el paciente, ni la concentración óptima, de tal forma que estimule la mayor proliferación en células del tejido conectivo como abroblastos u osteoblastos. Tales células son numerosas en el periodonto y son las responsables de la producción de la matriz orgánica de los tejidos que lo constituyen $[3,4,38,40]$. Los osteoblastos y los Abroblastos producen colágeno, el mayor componente de la matriz, y, por ello, están implicados en el proceso de regeneración tisular [7,13,41].

Acosta y colaboradores, en investigaciones publicadas en 2006 y 2010, observaron una tendencia al aumento en la proliferación celular hacia las 24 horas posteriores a la activación de PRP en Abroblastos gingivales $[42,43]$. Sin embargo, no se conoce la respuesta celular a diferentes tiempos después de su activación. Diversos estudios han evaluado el uso del PRP en diferentes concentraciones, que Áuctúan entre $0,05 \%$ y $100 \%[8,34,35,37,44,45]$, y sus resultados son heterogéneos. 
Por otra parte, también existe el plasma pobre en plaquetas (PPP), que tiene una concentración relativamente baja de plaquetas. El PPP se obtiene a partir de una primera centrifugación a una velocidad de $280 \mathrm{~g}(1400 \mathrm{rpm})$ durante 7 minutos o $160 \mathrm{~g}(1200 \mathrm{rpm})$ por 10 minutos. Con esta primera centrifugación, se consigue separar la sangre completa en una franja roja inferior de hematíes y una amarillenta superior de plasma [8].

El objetivo de este estudio fue evaluar la proliferación celular en líneas celulares de Abroblastos de ligamento periodontal y osteoblastos tratados con PRP y PPP, en concentraciones de 1\%, 3\% y 5\% a las 0, 12, 24, 48 y 72 horas después de su activación, con el propósito de conocer el tiempo y la concentración más efectivos para el uso clínico del PRP.

\section{MATERIALES Y MÉTODOS}

\section{Cultivo celular}

Se realizó un estudio experimental in vitro. Para el cultivo celular se utilizaron líneas celulares de Abroblastos de ligamento periodontal (Clonetics Human Fibroblast Cell System) y osteoblastos (Clonetics Normal Human Osteoblast) adquiridas de la casa comercial Lonza. Los Abroblastos se adquirieron en tercer pase y se sembraron en medio de cultivo Stromal Cell Basal (Clonetics ${ }^{\circledR}$ ) suplementado con suero fetal bovino (SFB) al $5 \%$, insulina al $0,1 \%$, factor de crecimiento Abroblástico básico al $0,1 \%$ y gentamicina/anfotericina $\mathrm{B}$ al $0,1 \%$. Los osteoblastos se adquirieron en segundo pase y se cultivaron en medio Normal Human Osteoblast Cell (Clonetics ${ }^{\circ}$ ) suplementado con SFB al 10\%, ácido ascórbico al 0,1\% y gentamicina/anfotericina B al 0,1\%. Ambos cultivos se llevaron a cabo siguiendo el protocolo de la casa fabricante. Posteriormente, al alcanzar entre $70 \%$ y $80 \%$ de conÁuencia, en cuarto pase para los Abroblastos y quinto pase para los osteoblastos, los cultivos celulares se tripsinizaron.

\section{Obtención y activación del PRP y el PPP}

El PRP y el PPP se obtuvieron de un donante voluntario hombre de edad 36 años, sistémicamente sano, que no había tomado antibióticos en los últimos 6 meses y no era fumador. El paciente aceptó hacer la donación mediante Arma del consentimiento informado, el cual fue aprobado por el Comité de Ética e Investigación de la Pontiacia Universidad Javeriana. Se utilizaron 6 tubos con ácido etilendiaminotetracético (EDTA), cada uno con $5 \mathrm{ml}$ de sangre venosa periférica extraída mediante punción de la vena cefálica en su unión con la vena mediana del codo de miembro superior. Se realizó un recuento de plaquetas electrónico de la muestra obtenida y se corroboró la Asiología sanguínea mediante tromboelastografía, con el an de evaluar que el conteo plaquetario y la Asiología sanguínea estuvieran entre los parámetros normales y así poder aceptar el donante. La centrifugación y separación del PRP y el PPP se realizó durante 7 minutos a $1400 \mathrm{rpm}[8,31,36]$. Posteriormente, se activaron los tubos con cloruro de calcio al 10\%, rotulados a diferentes tiempos después de la activación $(0,12,24,48$ y 72 horas) para su utilización. Una vez activados, los concentrados de PRP y $\mathrm{PPP}$ se mantuvieron almacenados a $-70^{\circ} \mathrm{C}$ para luego ser empleados en la prueba experimental. Se prepararon concentraciones al 1\%, al 3\% y al 5\% mediante diluciones con medio de cultivo con SFB, tanto del PRP como del PPP. 


\section{Evaluación de la viabilidad celular}

El tratamiento celular se realizó a cada una de las líneas descritas con PRP y PPP, al $1 \%$, al $3 \%$ y al 5 \% después de su obtención y activación a las $0,12,24,48$ y 72 horas para cada grupo. Se realizó el conteo celular basal en cámara de Neubauer y la siembra se llevó a cabo en cajas con $150 \mu \mathrm{l}$ (5000 células) por pozo. En cada caja se dejó un pozo de control en el cual se sembró medio con células sin tratamiento alguno y un pozo blanco con medio de cultivo exclusivamente. Para evaluar la viabilidad celular se aplicó el montaje y la prueba MTS, a efectos de lo cual se incubó por 3 horas a $37^{\circ} \mathrm{C}$, respetando los tiempos sugeridos por el proveedor, y luego se hizo la lectura de absorbancia mediante ensayo por inmunoabsorción ligado a enzimas (ELISA) a $490 \mathrm{~nm}$. Todas las pruebas se hicieron por triplicado.

\section{Análisis de los datos}

Los datos se recolectaron en una base de datos SPSS. El análisis de datos de absorbancia se realizó con el programa Sabiosciences ${ }^{\oplus}$, en tanto que para el análisis de los datos se aplicaron las pruebas $\mathrm{Chi}^{2}$, Fischer y $\operatorname{McNemar}(\mathrm{p}=0,05)$.

\section{RESULTADOS}

Se obtuvo el cultivo de Abroblastos y osteoblastos sin signos de contaminación hasta los pases descritos durante 12 días. El recuento de plaquetas obtenido de la sangre venosa del sujeto voluntario fue de 380.000/ $\mathrm{mm}^{3}$ y la curva de la tromboelastografía fue normal, lo que indica que había una Asiología normal apta para la preparación de PRP y PPP. Los resultados en el lector de ELISA se obtuvieron por triplicado y se promediaron los tres valores correspondientes a cada uno de los tratamientos realizados. A dicho valor se le restó el valor blanco y se establecieron porcentajes.

La Agura 1 ilustra la respuesta de los Abroblastos tratados con PRP y PPP a las 0, 12, 24, 48 y 72 horas, en concentraciones al $1 \%$, al 3\% y al $5 \%$ comparadas con el control. En el eje Y se observa que la absorbancia (nm) fue mayor para los Abroblastos estimulados con PRP al 5\% activados 24 horas después de su obtención. Con respecto al PPP, se advierte mayor absorbancia en el grupo control; sin embargo, ello coincide con la respuesta del PRP, en la que se muestra un aumento en la absorbancia en todos los grupos experimentales, en el especial en PPP al 5\% activado 24 horas después de su obtención.

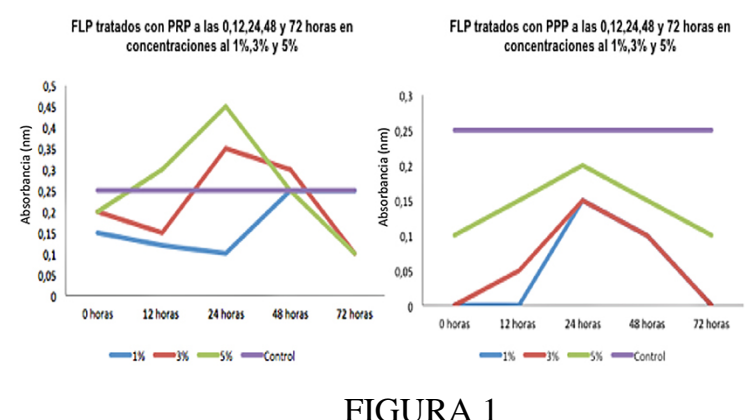

Fibroblastos tratados con PRP y PPP a las 0, 12, 24, 48 y 72 horas, en concentraciones al 1\%, al 3\% y al 5\% comparadas con el control

La Agura 2 ilustra la respuesta de los osteoblastos tratados con PRP y PPP a las 0, 12, 24, 48 y 72 horas, en concentraciones al 1\%, al 3\% y al 5\% comparadas con el control. Al igual que con los Abroblastos, se observa una mayor absorbancia en los osteoblastos estimulados con PRP y PPP al 5\% activados 24 horas después de su obtención. 

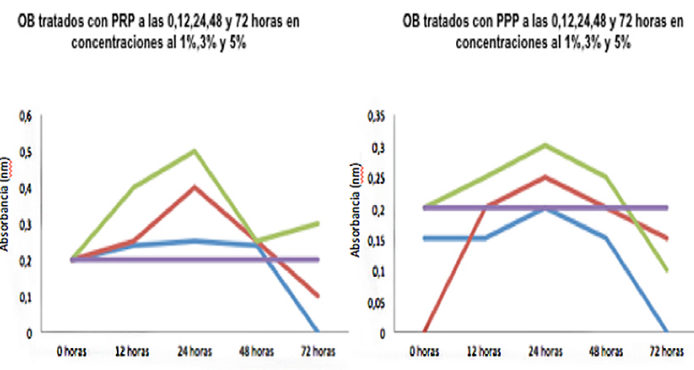

FIGURA 2

Respuesta de los osteoblastos tratados con PRP y PPP a las 0, 12, 24, 48 y 72

horas, en concentraciones al $1 \%$, al $3 \%$ y al $5 \%$, comparadas con el control

Al aplicar las pruebas estadísticas paramétricas, se evidencia que la diferencia fue estadísticamente signiAcativa con respecto a la obtención de una proliferación aumentada de Abroblastos con PRP al 5\% al aplicarlo 24 horas después de su procesamiento y activación. Las diferencias también fueron signiacativas para los osteoblastos $(\mathrm{p}=0,05)$.

\section{DISCUSIÓN}

El objetivo de este estudio fue evaluar la viabilidad celular observada en Abroblastos de ligamento periodontal y osteoblastos obtenidos mediante cultivo experimental y que se trataron con PRP a las 0, 12, 24, 48 y 72 horas luego de su procesamiento, en concentraciones de $1 \%, 3 \%$ y $5 \%$. En la literatura se han reportado diferentes protocolos de preparación después de la primera descripción del PRP como concentrado plaquetario de primera generación. Dohan y colaboradores [14] realizaron un recuento histórico desde las preparaciones que consistían en pegantes de Abrina hasta la obtención de concentrados plaquetarios de segunda generación como el plasma rico en factores de crecimiento (PRGF) y el plasma rico en Abrina (PRF). Así, observaron que las diferencias consisten en los componentes de Abrina y la presencia de leucocitos; sin embargo, no hay consenso en cuanto a su preparación, como se corrobora en las publicaciones de Anitua y colaboradores [18], Chang y Zhao [32], He y colaboradores [21] y Dohan y colaboradores [14,19, 20, 22, 25]. La ausencia de consenso es tal que Anitua y colaboradores [4] utilizan la sigla PRP en el título de su publicación y durante el desarrollo del texto se reaeren en forma reiterada al PRGF.

Por su parte, Balaram y colaboradores [46] describen métodos de obtención de derivados plaquetarios más simples si se comparan con la preparación del PRP. No obstante, los estudios disponibles aún no determinan la tasa de proliferación y viabilidad celular en humanos para aArmar cuál de los diferentes derivados sanguíneos, que involucran factores de crecimiento, tiene un impacto positivo en la regeneración celular [22, 25, 47]. He y colaboradores [21] comparan el efecto del PRF y el PRP sobre la proliferación celular y la diferenciación de osteoblastos en un modelo animal en ratas y cuantiacan los valores de PDGF-AB y TGF- $\beta 1$. Así, encuentran que el PRP libera grandes cantidades de estos factores durante el primer día, mientras que el PRF libera TGF- $\beta 1$ el día 14 y PDGF-AB el día 7 . Esto parece relacionarse con un efecto gradual y más duradero en la proliferación de los osteoblastos en el modelo utilizado.

En cuanto al uso del PRP en diferentes concentraciones, Kawase y colaboradores [35] lo utilizaron entre el $0,5 \%$ y el $2 \%$ para evaluar la síntesis de colágeno tipo I en células de ligamento periodontal y osteoblastos. En este sentido, reportaron que la preparación al 0,5\% se convertía en gel, al igual que preparaciones más concentradas. Fortes-Ferreira y colaboradores [48] utilizaron concentraciones de PRP progresivamente altas, a partir de 6,125\% y hasta 50\%, y observaron una mayor proliferación de osteoblastos mediante el uso de PRP al 50\%. Concentraciones similares utilizaron Slapnicka y colaboradores [37], quienes encontraron que concentraciones entre $10 \%$ y $25 \%$ se relacionan con mayor viabilidad celular. Beca y colaboradores [8] mencionan los estudios de Choi, en los cuales concentraciones entre el $1 \%$ y el $5 \%$ se relacionan con mayor 
viabilidad celular, lo cual coincide con lo evidenciado en el presente estudio. Mooren y colaboradores [34], después de utilizar PRP entre el $2 \%$ y el $8 \%$, aArman que no hay relación directa entre el número de plaquetas y los factores de crecimiento liberados a partir de ellas y que concentraciones más altas pueden generar apoptosis. Pantou y colaboradores [44] en su protocolo siempre lo preparan al $5 \%$ para su uso en investigación. Los anteriores estudios corroboran la heterogeneidad en el uso del PRP en cuanto a la concentración se reAere. Por lo tanto, los resultados de investigaciones realizadas in vitro que relacionen la utilización de PRP y la proliferación celular son difíciles de comparar [21]. En este estudio el proceso de preparación de los diferentes componentes utilizados y el cultivo celular fueron repetibles y predecibles, sin que se presentara ningún factor de sesgo. Ello minimizó el error al elaborarse por un mismo investigador. El aspecto, la respuesta y el crecimiento celular in vitro se corroboran con la respuesta de estas líneas celulares con la respuesta obtenida en diferentes investigaciones [22].

En este estudio se evidenció también una tendencia signiacativa a una mayor viabilidad celular deA broblastos y osteoblastos al utilizar PRP al 5\%, 24 horas después de su procesamiento y activación. Los resultados obtenidos sugieren que el PPP también modiaca la respuesta de viabilidad celular. Karp y colaboradores [49] utilizaron rellenos de Abrina como matrices en estudios de ingeniería tisular ósea en vivo. Su estudio, realizado en ratas, no mostró aumento en la invasión de tejido óseo. Esto sugiere que los factores de crecimiento podrían tener efectos diferentes de acuerdo con el derivado sanguíneo utilizado. Así se evidencia en el estudio de Æorwarth y colaboradores [50], quienes utilizaron colágeno bovino y PRP en un modelo experimental en cerdos domésticos. Estos investigadores en su análisis de inmunohistoquímica describieron la expresión de BMP-2, osteocalcina y osteonectina. Los estudios reportados por Stapicka y colaboradores y Forbes-Ferreira y colaboradores, realizados en osteoblastos, así como el estudio de Creeper y colaboradores, llevado a cabo adicionalmente en células del ligamento periodontal, utilizaron PRP inmediatamente después de su activación $[37,47,48]$. La respuesta positiva obtenida en el presente estudio sobre la viabilidad celular mediante el tratamiento con PPP coincide con los hallazgos de Dohan y colaboradores $[22,25]$. Dichos autores no encontraron diferencias estadísticamente signiacativas al cuantiA car las citoquinas contenidas en el PPP con respecto al PRP. No obstante, PDGF-BB y TGF- $\beta 1$ en el sobrenadante de PPP fueron signiacativamente menores que los obtenidos en el PRP. De esta manera, el PPP puede tener algún efecto menor comparado con el efecto del PRP.

En la literatura disponible no se reporta la respuesta de viabilidad de células periodontales tratadas con PRP a diferentes tiempos posteriormente a su activación. He y colaboradores [21] mencionan que los factores de crecimiento se liberan en las primeras 24 horas y utilizan principalmente el PRP inmediatamente después de su activación $[8,25,30,31,34,35,36,37,48,51]$. Los estudios de Acosta y colaboradores publicados en 2006 y 2010 evidencian una tendencia en la respuesta de viabilidad en A broblastos gingivales, los cuales presentan diferencias con respecto a los Abroblastos [42, 43]. Este hallazgo, sumado al resultado del presente estudio, sugiere que el efecto del PRP no es de tipo celular-especíaco y en la evidencia cientíaca disponible no hay comparaciones del tratamiento con PRP en diferentes tiempos luego de su activación. Por ello, los resultados obtenidos son innovadores en el campo de la biotecnología. Sobre esta base, el grupo investigador propone continuar la investigación del PRP y PPP in vitro e in vivo, con el An de proporcionar un fundamento racional a su utilización y no solo basarla en la experiencia de los clínicos.

\section{CONCLUSIONES}

El efecto del PRP al 5\% sobre Abroblastos y osteoblastos mostró una tendencia a mayor viabilidad celular en el tratamiento 24 horas después de su activación, lo cual puede tener una aplicación clínica para investigaciones in vivo. 


\section{RECOMENDACIONES}

Se sugiere realizar estudios comparados entre los diferentes protocolos de preparación, estudiar la expresión génica y estandarizar su obtención y preparación, con el An de promover la óptima utilización a los derivados sanguíneos de este tipo.

\section{REFERENCIAS}

1. Aljehani YA. Risk factors of periodontal disease: review of the literature. Int J Dent. 2014; 2014: 182513. doi: $10.1155 / 2014 / 182513$.

2. Armitage GC, Robertson PB. Æe biology, prevention, diagnosis and treatment of periodontal disease: scientiac advances in the United States. J Am Dent Assoc. 2009; 140(Suppl 1): 365-435.

3. Dentino A, Lee S, Mailhat J, Hexi A. Principles of periodontology. Periodontol 2000. 2013 Feb; 61(1): 16-53.

4. Anitua E, Troya M, Orive G. An autologous platelet rich plasma stimulates periodontal ligament regeneration. J Periodontol. 2013 Nov; 84(11): 1556-66.

5. García-Martínez O, Reyes-Botella C, Díaz-Rodríguez L, Luna-Bertos E, Ramos-Torrecillas J, Vallecillo-Capilla M, Ruiz C. Effect of platelet-rich plasma on growth and antigenic proAle of human osteoblasts and its clinical impact. J Oral Maxillofac Surg. 2012; 70: 1558-64.

6. Rios H, Lin Z, Oh B, Park Ch, Giannobile W. Cell and gene-based therapeutic strategies for periodontal regenerative medicine. J Periodontol. 2011; 82: 1223-37.

7. Hallman M, Æo $\mathrm{r}$ A. Bone substitutes and growth factors as an alternative/complement to autogenous bone for graÄing in implant dentistry. Periodontol 2000. 2008 Jun; 47(1): 172-92.

8. Beca T, Hernández G, Morante $S$, Bascones A. Plasma rico en plaquetas. Una revisión bibliográAca. Avances en Periodoncia. 2007 Abr; 19(1): 39-52.

9. Hughes FJ, Turner W, Belibasakis G, Martuscelli G. Effects of growth factors and cytokines on osteoblast differentiation. Periodontol 2000. 2006 Jun; 41(1): 48-72.

10. Polimeni G, Xiropaidis AV, Wikesjö UME. Biology and principles of periodontal wound healing/regeneration. Periodontol 2000. 2006 Jun; 41(1): 30-47.

11. Cochran DL, Wozney JM. Biological mediators for periodontal regeneration. Periodontol 2000. 1999 Feb; 19(1): 40-58.

12. Souren J, Van Wijk R. Fibroblastic cell cycling in collagen gels. Cell Proliferation. 1993 Jan; 26(1): 12-23.

13. Acosta A. Papel del Abroblasto en salud y enfermedad periodontal. En: Gutiérrez S, editora. Fundamentos de ciencias básicas aplicadas a la odontología, primera edición. Bogotá, Colombia: Pontiacia Universidad Javeriana; 2006. pp. 349-68.

14. Dohan D, Andia I, Zumstein M, Zhang Ch, Pinto N, Bielecki T. ClassiAcation of platelet concentrates (PlateletRich Plasma- PRP, Platelet- Rich Fibrin- PRF) for topical and inAltrative use in orthopedic and sports medicine: current consensus, clinical implications and perspectives. Muscles, Ligaments and Tendons J. 2014; 4(1): 3-9.

15. Lee JW, Kwon OH, Kim TK, Cho YK, Choi KY, Chung HY, Cho BC, Yang JD, Shim JH. Platelet-rich plasma: quantitative assessment of growth factor levels and comparative analysis of activated and inactivated groups. Arch Plast Surg. 2013; 40: 530-5.

16. Carrillo-Mora P, González-Villalta A, Macías-Hernández S, Pineda-Villaseñor C. Plasma rico en plaquetas. ¿Herramienta versátil de la medicina regenerativa? Cir Cir. 2013; 81: 74-82.

17. Burnouf T, Goubran H, Che TM, Ou KL, El-Ekiaby M, Radosevic M. Blood-derived biomaterials and platelet growth factors in regenerative medicine. Blood Reviews. 2013; 27: 77-89. 
18. Anitua E, Troya M, Orive G. Plasma rich in growth factors promote gingival tissue regeneration by stimulating Abroblast proliferation and migration and by blocking transformin growth factor $\beta$ - 1 induced myodifferentiation. J Periodontol. 2012; 83(8): 1028-37.

19. Dohan D. How to optimize the preparation of leukocyte and platelet- rich Abrin (L-PRF, Choukroun's technique) clots and membranes: Introducing the PRF Box. Oral Surg Oral Med Oral Pathol Oral Radiol Endod. 2010 Sep; 110(3): 275-8.

20. Dohan D. Platelet- rich Plasma (PRP) and platelet rich Abrin (PRF) in human cell cultures: Growth factor release and contradictory results. Oral Surg Oral Med Oral Pathol Oral Radiol Endod. 2010; 110(4): 418-21.

21. He L, Lin Y, Hu X, Zhang Y, Wu H. A comparative study of platelet-rich Abrin (PRF) and platelet- rich plasma (PRP) on the effect of proliferation and differentiation of rat osteoblast in vitro. Oral Surg Oral Med Oral Pathol Oral Radiol Endod. 2009; 108(5): 707-13.

22. Dohan D, Rasmusson L, Albreksson T. Classiacation of platelet concentrates: from pure platelet-rich plasma (PPRP) to leucocyte and platelet-rich Abrin (LPRF). Trends Biotechnol. 2009; 27(3): 158-67.

23. Nikolidakis D, Jansen J. «e biology of platelet- rich plasma and its application in oral surgery: Literature review. Tissue Eng Part B Rev. 2008; 14(3): 249-58.

24. Han J, Meng HX, Tang JM, Chen ZB. Æe effect of different platelet-rich plasma concentration on proliferation of human periodontal ligament cells in vitro. Cell Proliferation. 2007 Apr; 40(2): 241-52.

25. Dohan D, Choukroun J, Diss A. Platelet-rich Abrin (PRF): A second-generation platelet concentrate. Part I: technological concepts and evolution. Oral Surg Oral Med Oral Pathol Oral Radiol Endod .2006; 101(3): e37-44.

26. Zhang N, Wu YP, Quian SJ, Teng Ch, Chen Sh, Li H. Research progress in the mechanism of effect of PRP in bone deAciency healing. Scientiac World J. 2013 Apr 4: 1-7.

27. Saucedo JM, Yaffe MA, Berschback JC, Hsu WK, Kalainov DM. Platelet-rich plasma. J Hand Surg Am. 2012; 37(3): 587-9.

28. Cervelli V, Scioli M, Gentile P, Doldo E, Bonanno E, Spagnoli L, Orlandi A. Platelet-rich plasma greatly potentiates insulin-induced adipogenic differentiation of human adipose-derived stem cells through a serine threonine kinase akt-dependent mechanism and promotes clinical fat gra é maintenance. Stem Cells Transl Med. 2012; 1(3): 206-20.

29. Gentile P, Bottini D, Spallone D, Curcio B, Cervelli V. Application of PRP in maxillofacial surgery. J Craniofac Surg. 2010; 21: 900-4.

30. Froum S, Wallace S, Tarnow D, Cho SC. Effect of PRP on bone growth in maxillary sinus graÁs. A ree bilateral case reports. Int J Periodontics Restorative Dent. 2002; 22: 45-53.

31. Marx R, Carlson E, Eichstaedt R, Schimmele S, Strauss J, Georgeff K. Platelet-rich plasma- Growth factor enhancement for bone gra As. Oral Surg Oral Med Oral Pathol Oral Radiol Endod. 1998; 85: 638-46.

32. Chang YC, Zhao JH. Effects of platelet-rich Abrin on human periodontal ligament Abroblasts and application for periodontal infrabony defects. Australian Dental J. 2011; 56:365-71.

33. Plachokova A, Nikolidakis D, Mulder J, Jansen J, Creugers N. Effect of Platelet-Rich Plasma on bone regeneration in dentistry: a systematic review. Clin Oral Impl Res. 2008; 19: 539-45.

34. Mooren R, Hendriks E, van den Beaken J, Merkx M, Meijer G, Jansen J, Stoelinga PJ. Æe effect of platelet-rich plasma in vitro on primary cells rat osteoblast-like cells and human endotelial cells. Tissue Eng Part A. 2010; 16(10): 3159-72.

35. Kawase T, Okuda K, Wolff L, Yoshie H. Platelet-rich plasma-derived Abrin clot formation stimulates collagen synthesis in periodontal ligament and osteoblast cells in vitro. J Periodontol 2003; 74: 858-64.

36. Gonshor A. Technique for producing PRP and platelet concentrate: Background and process. Int J Periodontics Rest Dent. 2002; 22: 547-57.

37. Slapnicka J, Frassmann A, Strasak L, Augustin P, Vanek J. Effects of activated and nonactivated platelet-rich plasma on proliferation of human osteoblast in vitro. J Oral Maxillofac Surg. 2008; 66: 297-301. 
38. Reyes M, Montero S, Cifuentes J, Zarzar E. Actualización de la técnica de obtención y uso del plasma rico en factores de crecimiento (PRGF). Rev Dental Chile. 2002; 93(2): 25-8.

39. Sodek J, McKee M. Molecular and cellular biology of alveolar bone. Periodontol 2000. 2000; 24: 99-126.

40. Demmer RT, Papapanou PN. Epidemiologic patterns of chronic and aggressive periodontitis. Periodontol 2000. 2010 Jun; 53(1): 28-44.

41. Anitua E, Sanchez M, Zalduendo M, De La Fuente M, Prado R, Orive G, Andia I. Fibroblastic response to treatment with different preparations rich in growth factors. Cell Proliferation. 2009 Apr; 42(2): 162-70.

42. Acosta A, González O, Pereira E, Sánchez A. Proliferación de Abroblastos gingivales humanos tratados con plasma rico en plaquetas in vitro. Rev Fundación Juan José Carraro (Argentina). 2006 Abr-May; (22): 8-14.

43. Acosta A, García D, Perdomo S, González O. Efecto del plasma rico en plaquetas sobre la proliferación de Abroblastos gingivales humanos in vitro. Rev Fundación Juan José Carraro (Argentina). 2010 Abr-May; (31): 4-13.

44. Pantou AL, Markopoulo CE, Dereka XE, Vavoraki H, Mamalis A, Vrotsos IA. «e effect of platelet-rich plasma (PRP) combined with a bone allograte on human periodontal ligament (PDL) cells. Cell Tissue Bank. 2012; 13: 81-8.

45. Markopoulou CE, Markopoulou P, Dereka XE, Pepelass E, Vrotsos IA. Effect of homologous PRP on proliferation of human periodontally affected osteoblast. In vitro preliminary study. Report a case. J Musculoskelet Neuronal Interact. 2009; 9(3): 167-72.

46. Balaram N, Karunakar P, Jayadev M, Marshal R. Role of platelet rich Abrin in wound healing: A critical review. J Conserv Dent. 2013; 16(4): 284-93.

47. Creeper F, Lichanska AM, Marshall RI, Seymour GJ, Ivanovski S. Æe effect of platelet-rich plasma on osteoblast and periodontal ligament cell migration, proliferation and differentiation. J Periodont Res. 2009; 44: 258-65.

48. Fortes-Ferreira C, Carriel M, Filho J, Granjeiro J, Oliveira C, Magini R. Platelet-rich plasma ináuence on human osteoblasts growth. Clin Oral Impl Res. 2005; 16: 456-60.

49. Karp JM, Sarraf F, Schoichet MS, Davies JE. Fibrin-Alled scaffolds for bone-tissue engineering: an in vivo study. J Biomed Mater Res A. 2004; 71(1): 162-71.

50. Æ orwarth M, Rupprecht S, Falk S, Felszeghy E, Wiltfang J, Schlegel KA. Expression of bone matrix proteins during de novo bone formation using a bovine collagen and platelet-rich plasma (PRP) an immunohistochemical analysis. Biomaterials. 2005; 26: 2575-84.

51. Tsay RC, Vo J, Burke A. Differential growth factor retention by platelet-rich plasma composites. J Oral Maxillofac Surg. 2005; 63: 521-8.

\section{Licencia Creative Commons CC BY 4.0}

Cómo citar este artículo: Acosta AP, Gutiérrez SJ, Bedoya MA, García DA, Moreno XE. Evaluación del efecto del plasma rico en plaquetas en diferentes tiempos y concentraciones sobre la proliferación de abroblastos de ligamento periodontal y osteoblastos. Univ Odontol. 2017 Ene-Jun; 36(76): 1-9. https://doi.org/10.11144/Javeriana.uo36-76.eepr 